\title{
Cytotoxicity Comparison of the Nanoparticles Deposited on Latex Rubber Bands between the Original and Stretched State
}

\author{
Jung-Hwan Lee, ${ }^{1,2}$ Eun-Jung Lee, ${ }^{1}$ Jae-Sung Kwon, ${ }^{1}$ \\ Chung-Ju Hwang, ${ }^{3}$ and Kyoung-Nam Kim ${ }^{1,2}$ \\ ${ }^{1}$ Department and Research Institute of Dental Biomaterials and Bioengineering, Yonsei University College of Dentistry, \\ 50-1 Yonsei-ro, Seodaemun-gu, Seoul 120-752, Republic of Korea \\ ${ }^{2}$ BK21 PLUS Project, Yonsei University College of Dentistry, 50-1 Yonsei-ro, Seodaemun-gu, Seoul 120-752, Republic of Korea \\ ${ }^{3}$ Department of Orthodontics and The Institute of Cranio-Facial Deformity, Yonsei University College of Dentistry, \\ 50-1 Yonsei-ro, Seodaemun-gu, Seoul 120-752, Republic of Korea
}

Correspondence should be addressed to Chung-Ju Hwang; hwang@yuhs.ac and Kyoung-Nam Kim; kimkn@yuhs.ac

Received 19 May 2014; Accepted 14 July 2014; Published 7 August 2014

Academic Editor: Seunghan Oh

Copyright () 2014 Jung-Hwan Lee et al. This is an open access article distributed under the Creative Commons Attribution License, which permits unrestricted use, distribution, and reproduction in any medium, provided the original work is properly cited.

Understanding the biocompatibility of nanoparticles in dental materials is essential for their safe usage in the oral cavity. In this study, we investigated whether nanoparticles deposited on orthodontic latex rubber bands are involved in the induction of cytotoxicity. A method of stretching to three times ("3L") the length of the latex rubber bands was employed to detach the particles using the original length ("L") for comparison. The cytotoxicity tests were performed on extracts with mouse fibroblasts (L929) and human gingival fibroblasts (HGFs). Fourier transform infrared spectroscopy, ion chromatography, elemental analysis, and inductively coupled plasma mass spectrometry (ICP-MS) were performed to detect the harmful components in the extracts from rubber bands. There was a significant decrease in the cell viability in the "L" samples compared with the "3L" samples $(P<0.05)$ in the L929 and HGF cells. This was due to the Ni single crystal nanoparticles ( 50nm) from the inner surface of "L" samples that were detached in the "3L" samples as well as the $\mathrm{Zn}$ ion $(\sim 9 \mathrm{ppm})$ detected in the extract. This study revealed that the Ni nanoparticles, as well as $\mathrm{Zn}$ ions, were involved in the induction of cytotoxicity from the latex rubber bands.

\section{Introduction}

Nanomaterials offer significant promise for a variety of dental applications, including tooth scanning, the prevention of tooth decay, and as a component of the biomaterials used to enhance the mechanical and antiwear properties [1-3]. However, before these nanomaterials can become a clinical reality, the toxicity and biocompatibility of the nanoparticles must be carefully evaluated to reduce the adverse biological responses $[4,5]$. Therefore, understanding the biocompatibility of the nanoparticles deposited on dental materials is essential for safe usage in the oral cavity.

Latex rubber bands are commonly used in orthodontic treatments to apply a certain force to the teeth, although latex-related disease has become a concern for those using the latex-containing products [6-8]. Sulfur and zinc oxide particles, used as preservatives, and nickel, used as an accelerator, have been shown to be cytotoxic [9]. However, the nanoparticles on latex rubber bands have not been recognized as a potential cytotoxic ingredient despite their deposition on the surface.

Numerous studies have been performed to evaluate the in vitro cytotoxicity of orthodontic elastic materials, including bands, separators, and ligatures [9-13]. Those reports have shown that latex elastomeric materials show cytotoxicity in vitro, typically using a cell viability test. However, discerning the biological side effects from the latex elastomeric materials in orthodontic patients has been difficult. According to more than 30 years of clinical experience, patients have only rarely suffered a harmful event caused by latex rubber bands used in a clinical application (e.g., oral lesion, gastric problem, and other local symptoms). In summary, the previous in vitro 
cytotoxicity tests did not appear to reflect the clinical results gathered by the careful observations of clinicians. This type of inconsistency between the clinical and in vitro results has been commonly observed in other studies [14]. Therefore, an effort to reveal the reason for the rare but harmful events from the dental materials in the human oral cavity due to the reported cytotoxicity in in vitro studies is required to decrease the inconsistency. In this study, the role of the nanoparticles deposited on the latex rubber bands in terms of cytotoxicity was elucidated to decrease the above inconsistency.

To evaluate the latex rubber bands, in vitro cytotoxicity tests were performed according to the ISO 21606 and ISO 10993-5 and 12 standards [15-17]. According to ISO 1099312 , the extraction conditions should attempt to simulate the clinical use conditions to determine the potential toxicological hazard without causing significant changes. However, to date, the in vitro cytotoxicity tests on latex rubber bands reported in the orthodontic literature have been performed without a serial dilution of the extracts and without any stretching or compressing of the materials $[9,11,13,18]$, even though the latex rubber bands are used in contact with oral mucosa and saliva in a stretched state of up to three times their length ("3L”) [17]. Moreover, mouse fibroblast (L929) cells have typically been used to evaluate the cytotoxicity of latex elastomeric materials, which are less reflective of the response of human oral cells against the harmful extracts from the latex rubber bands. Therefore, studies that mimic the manner in which the elastomeric materials are used in the oral cavity have been performed along with the reevaluation of the cytotoxicity of the dental materials in situations that mimic their clinical use [18-22].

Hence, we investigated whether the nanoparticles deposited on orthodontic latex rubber bands are involved in inducing cytotoxicity. To compare the cytotoxicity induced by the nanoparticles, the latex rubber bands were stretched to three times (" $3 \mathrm{~L}$ ") their original length, and the original length ("L") was used as the control group. The cytotoxicity tests were performed with mouse fibroblasts (L929) and human gingival fibroblasts (HGFs).

\section{Materials and Methods}

2.1. Materials. Latex rubber bands from three different manufacturers were selected (Table 1). The "L" sample as is and the " $3 \mathrm{~L}$ " sample after being stretched using a rectangular titanium (Ti, Buehler, Lake Bluff, IL, USA) piece were prepared for immersion in extracting media and visualization by scanning electron microscopy (SEM). The Ti was cut to the original length or three times the length of the rubber band. When the "L" sample was incubated in the extracting media, an equal amount of titanium to that used in the "3L" sample was also immersed. All the materials were treated with ethylene oxide for sterilization and were exposed to air for $48 \mathrm{~h}$ to eliminate the remaining gas.

2.2. Tests on the Extracts and Cell Viability. The tests on the extracts were performed according to ISO 10993-12 [16]. The extracts were prepared from the "3L" or "L" latex rubber bands in each well of standard 6-well plates (SPL, Pocheon, Korea)
TABLE 1: Summary of the materials used.

\begin{tabular}{lccc}
\hline Name & Code & Main composition & Manufacturer \\
\hline Giant Panda & GP & Natural latex & American Orthodontics, \\
Unitek & UN & Natural latex & USA \\
Extream & EX & Natural latex & ODP, USA \\
\hline
\end{tabular}

containing 3.5 mL of RPMI-1640 (Welgene, Daegu, Korea) for the L929 cells or DMEM (Welgene, Daegu, Korea) for the HGFs. The L929 cells (mouse fibroblast, NCTC clone 929, Korean Cell Line Bank, Korea) or HGFs [23] (HGF-1, CRL2014, ATCC, USA) were plated at a concentration of $1 \times 10^{4}$ cells per well in standard 96-well plates (SPL, Korea) in $100 \mu \mathrm{L}$ of culture medium and incubated at $37^{\circ} \mathrm{C}$. Following $24 \mathrm{~h}$ of cell culture, the monolayer was exposed to $100 \mu \mathrm{L}$ of extract or fresh medium (the control) for $24 \mathrm{~h}$. The $100 \%$ extracts were serially diluted to $50 \%, 25 \%, 12.5 \%$, and $6.25 \%$. Each diluted extract was added to one well and incubated at $37^{\circ} \mathrm{C}$ under relatively humidified conditions. The WST solution $(10 \mu \mathrm{L})$ was added to each well, and the cells were incubated for $3 \mathrm{~h}$ to allow the formation of formazan crystals, which were measured at $450 \mathrm{~nm}$ with a microplate spectrophotometer (BioTek, Winooski, Vermont, USA). Six wells were used to test each condition, and the experiments were performed in triplicate.

2.3. Agar Diffusion Test. The agar diffusion test was conducted based on the procedures described by ISO 109935 [15]. The L929 monolayer was overlaid with agar stained with a vital dye (neutral red), which allows diffusion of the leachable chemicals from the specimen. The " $3 \mathrm{~L}$ " and " $\mathrm{L}$ " samples and the positive (natural latex) and negative (high density polyethylene sheet) controls were positioned on the solidified agar layer. Prestretched rubber bands from the " $3 \mathrm{~L}$ " group and unstretched rubber bands from the "L" group were used for the direct agar diffusion test. After a $24 \mathrm{~h}$ incubation under the appropriate cell culture conditions, the biological reactivity (i.e., cellular degeneration and malformation) was rated on a scale of grade 0 (no reactivity) to grade 4 (severe reactivity) according to the zone extending from the specimen. The test was performed in triplicate.

2.4. Surface and Extract Characterization. To visualize the surface texture of the elemental composition from the inner and outer morphology of the "L" and "3L" samples, SEM (FE SEM S-800, Hitachi, Japan) with energy dispersive spectroscopy (EDS, Oxford Instruments, UK) was used. The detailed structural properties of the detached nanoparticles were investigated by a high resolution transmission electron microscopy (HR-TEM, JEM 3010) with EDS at an accelerating voltage of $300 \mathrm{kV}$ and selected area electron diffraction (SAED). Fourier transform infrared spectrometry (FT-IR, Vertex70, Bruker, Germany) with an attenuated total reflectance (ATR) was used to detect the harmful functional groups in the extracts from the "L" and "3L" samples. Each extracted solution was placed on the crystal surface of the ATR device and examined. 
2.5. Ion Chromatography, Elemental Analysis, and Inductively Coupled Plasma Mass Spectrometry. To measure the possible harmful ions of the extracts, the major anions $\left(\mathrm{F}, \mathrm{Cl}, \mathrm{Br}, \mathrm{NO}_{2}\right.$, $\mathrm{NO}_{3}, \mathrm{PO}_{4}$, and $\mathrm{SO}_{4}$ ) were analyzed by ion chromatography (Dionex Model ICS-2000, USA). To evaluate the quantity of the elemental contents from the extract, the $\mathrm{C}, \mathrm{H}, \mathrm{N}$, and S contents were measured with a 2400 Series II CHNS/O element analyzer (Perkin Elmer, USA). Inductively coupled plasma mass spectrometry (ICP-MS) was used to measure the harmful elements, such as $\mathrm{Zn}, \mathrm{Ni}, \mathrm{Fe}, \mathrm{Mg}$, and $\mathrm{Cu}$, in the extracts of the "L" and "3L" samples. The evaluation was performed at least three times.

2.6. Statistics. The statistical analyses comparing the "L" and "3L" samples were performed by the independent $t$-test using the SPSS PASW 18.0 program (SPSS Inc., Chicago, IL, USA). The significance was set at $P<0.05,0.01$, or 0.001 , depending on the circumstance. Representative results or images are shown after the experiments were performed in at least triplicate.

\section{Results}

The results of the cell viability test following the exposure to the extracts of the "L" and " $3 \mathrm{~L}$ " latex rubber bands are shown in Figures 1 and 2. In terms of cytotoxicity for the L929 cells, the Extream- (EX-) "3L" sample showed significantly lower cell viability compared with the EX-"L" sample when the L929 cells were exposed to the 50\% extracts (Figure 1(b), $P<0.01)$, and the cell viability of the $12.5 \%$ extracts of the Giant Panda- (GP-) "L" and Unitek- (UN-) "L" samples was significantly lower $(P<0.05)$ compared with that of the $12.5 \%$ extracts of the "3L" sample for each test group (Figure 1(c)). When the "L" sample was used for the cytotoxicity test, the acceptable cell viability (more than $70 \%$ ) of the extract was determined to be at a $6.25 \%$ dilution for all brands (Figure $1(\mathrm{~d}))$. However, when the " $3 \mathrm{~L}$ " sample was used, the $12.5 \%$ dilution achieved the acceptable viability level (Figure 1(c)). When the HGFs were used for the cytotoxicity test, the three brands showed significant differences between the "L" and "3L" samples (Figure 2(b)) for the $25 \%$ extracts.

The agar diffusion test was performed to confirm the difference in the cytotoxicity. Overall, the GP, UN, and EX rubber bands had moderate cytotoxicity (score 3 ), displaying a zone extending up to $1.0 \mathrm{~cm}$ around the specimen boundary (Figure 3). However, the EX group had a relatively smaller cell lysis zone compared with the GP and UN groups.

The FT-IR results indicated increased IR-transmittance at 1020 (C-O stretch), 2850, and $2917 \mathrm{~cm}^{-1}$ (C-H stretch) compared with the DMEM culture media control group (Figure 4). According to the SEM images in Figures 5 and 6, when the latex rubber bands are stretched to the " $3 \mathrm{~L}$ " position, the numbers of micro- and nanoparticles deposited on the inner surface compared were reduced with the "L" samples. However, on the outer surface, there were no particles, and the crevice was only detected in the "3L" configuration (Figure 5). C, O, S, and other elements were detected in the micro- (right headed white arrow) and nano- (left headed white arrow with a rectangle) particles in Figure 6. The
TABLE 2: ICP-MS results of the latex rubber bands extract.

\begin{tabular}{lcc}
\hline Sample & Zn $(\mathrm{ppm})$ & $\mathrm{Ni}(\mathrm{ppm})$ \\
\hline GP-L & $8.45 \pm 0.13^{\mathrm{a}, \mathrm{b}}$ & $\mathrm{ND}$ \\
GP-3L & $7.95 \pm 0.08^{\mathrm{a}}$ & $\mathrm{ND}$ \\
UN-L & $7.88 \pm 0.21^{\mathrm{a}}$ & $\mathrm{ND}$ \\
UN-3L & $7.66 \pm 0.14^{\mathrm{a}}$ & $\mathrm{ND}$ \\
EX-L & $7.53 \pm 0.13^{\mathrm{a}, \mathrm{b}}$ & ND \\
EX-3L & $5.78 \pm 0.05^{\mathrm{a}}$ & ND \\
Control & $0.24 \pm 0.01$ & ND \\
\hline
\end{tabular}

Concentration of the "L" and " $3 \mathrm{~L}$ " samples from the GP, UN, and EX extracts.

${ }^{\mathrm{a}, \mathrm{b}} P<0.05$; ${ }^{\mathrm{a}}$ compared with the control and ${ }^{\mathrm{b}}$ compared with the $3 \mathrm{~L}$ sample in each product. ND: not detected.

clearing of Ni was shown for the nanoparticles in the "3L" configuration but not in the "L" configuration; in contrast, no significant change in the other elements was observed (Figure 6(a) versus Figure 6(b)). The SEM images under low magnification showed that the adherent particles and the powder in the " $\mathrm{L}$ " group were significantly attached compared with those in the "3L" group (Figure 7(a) versus 7(d), 7(b) versus $7(\mathrm{e})$ and $7(\mathrm{c})$ versus $7(\mathrm{f})$ ). The EDS mapping analysis showed that the inner surface of all the "L" samples contained $\mathrm{Ni}$ nanoparticles but that none of the "3L" samples contained $\mathrm{Ni}$ (Figures $7(\mathrm{~g}), 7(\mathrm{~h})$, and $7(\mathrm{i})$; the Ni element in "3L" was not shown due to its absence). The detached Ni nanoparticles from the GP group, with sizes of $\sim 50 \mathrm{~nm}$, are representatively shown in the TEM images in Figure 8(a), and the presence of $\mathrm{Ni}$ is confirmed by EDS in Figure 8(b). The SAED dot patterns imply the single crystalline nature of the Ni single crystal, from which the (111) plane can be indexed in Figures 8(c) and $8(\mathrm{~d})$ (d-spacing: $0.20 \mathrm{~nm}$ ) [24,25]. However, the Ni ions in the extracts were undetected in the "L" and "3L" groups of all of the products, indicating that the detached Ni nanoparticles were not ionized in the media and, consequently, could not be detected by ICP-MS (Table 2). The ICP-MS results only indicated that the " $3 \mathrm{~L}$ " and "L" extracts showed an increased concentration of $\mathrm{Zn}$ ions compared with the control media and that the "3L" extract showed a smaller concentration of $\mathrm{Zn}$ ions, a component of latex rubber preservatives, compared with the "L" extract (Table 2). The results of the ion chromatography and elemental analyses did not show a significant difference among the experimental groups, except for the sulfate and fluoride contents between the U-L and U$3 \mathrm{~L}$ groups (Tables 3 and $4, P>0.05$ ).

\section{Discussion}

Increased concern about the toxicity of nanoparticles has occurred in dentistry due to their dental application, which encompasses the powders for scanning the oral anatomy, including tooth and gingiva, the ingredients of the preventive restorative materials used against tooth decay, and the supplemental nanoparticles (e.g., amorphous silicon dioxide and ground glass particle) in the filling materials [26]. In addition to the above intended purposes, nanoparticles were unintentionally found in other dental materials or during the grinding and polishing processes for the filling materials $[27,28]$. 


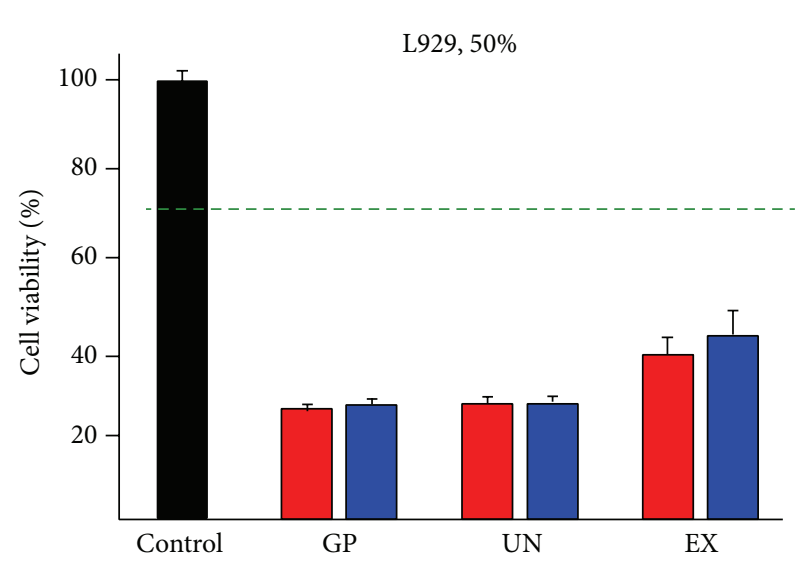

(a)

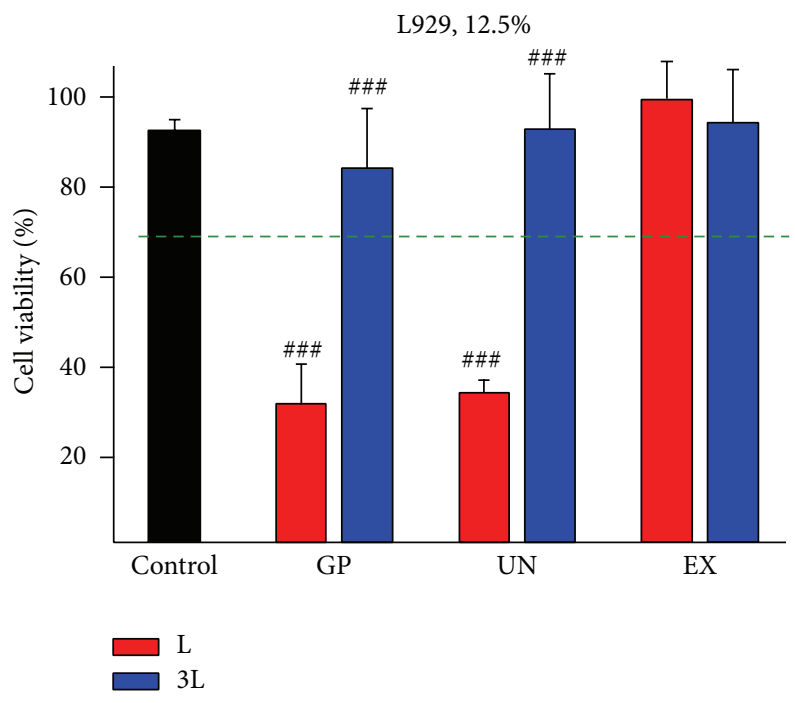

(c)

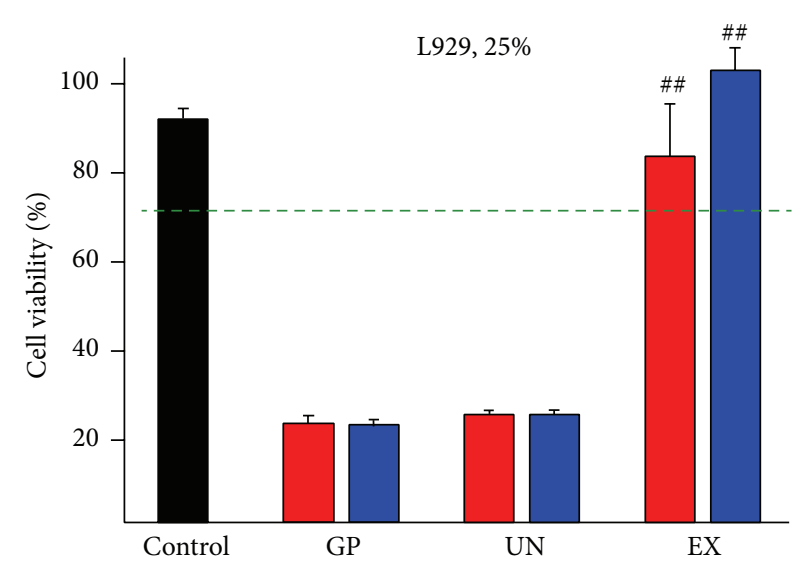

(b)

L929, 6.25\%

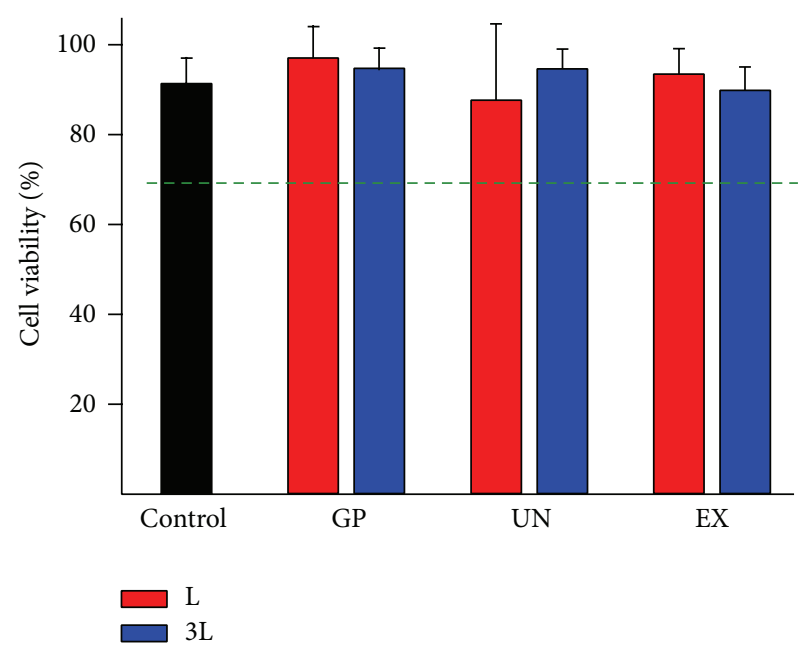

(d)

Figure 1: L929 cell viability following exposure to the extracts from the "3L" and "L" latex rubber bands of the Giant Panda (GP), Unitek (UN), and Extream (EX) extracts. The following dilutions were used: (a) 50\%, (b) 25\%, (c) 12.5\%, and (d) $6.25 \%$. The difference in the cell viability was statistically determined under a few experimental conditions $\left(n=6,{ }^{\# \#} P<0.01,{ }^{\# \# \#} P<0.001\right.$; between the "3L" and "L" extracts of each evaluated product). L: original length, 3L: stretched to three times its length. Representative results are shown after the experiments were performed in triplicate.

The presence of nanoparticles in these dental materials has raised concern regarding whether these dental material nanoparticles could be released and cause adverse health risks to humans. In this study, a conventionally used elastomeric orthodontic material, latex rubber bands, was used to evaluate the cytotoxicity induced by the deposited nanoparticles.

Latex rubber bands are widely used in orthodontic treatments and are considered medical devices in the mouth; thus, they require a series of safety evaluations for use in patients. Using in vitro tests, the cytotoxicity of latex rubber bands has been revealed in many studies $[9,10]$. However, latex rubber bands have been safely used in patients without an allergy to latex, which was explained due to the dilution effect from the saliva in the oral cavity during their use. In this study, nanoparticles were found on the inner surface of the latex rubber bands, and those nanoparticles were considered to be a potential inducer of cytotoxicity. Therefore, the purpose of this experiment was not to rank or reevaluate the cytotoxicity of latex rubber bands but to identify the nanoparticleinduced cytotoxicity by comparison of the cytotoxicity results associated with stretched latex rubber bands, which cause detachment of the deposited nanoparticles from the latex rubber bands.

Previously, the cytotoxicity tests for orthodontic elastic materials have used different diluted extracts of nonstretched materials, which does not appear to consider the presence of nanoparticles on the orthodontic latex rubber band [9-12]. In this study, latex rubber bands that were stretched to three times their length (" $3 \mathrm{~L}$ ") were used to detach the deposited particles and showed a significantly decreased cytotoxicity compared with the nonstretched materials ("L") under the specific diluted conditions in L929 cells and in HGFs (Figures 1 and 2). However, in the direct agar diffusion test, the "L" and "3L" groups showed moderate cytotoxicity (Figure 3), with 


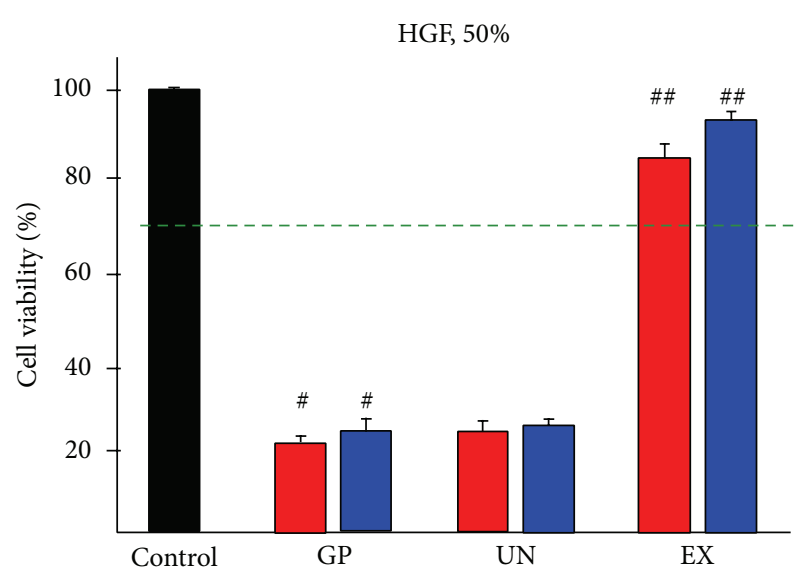

(a)

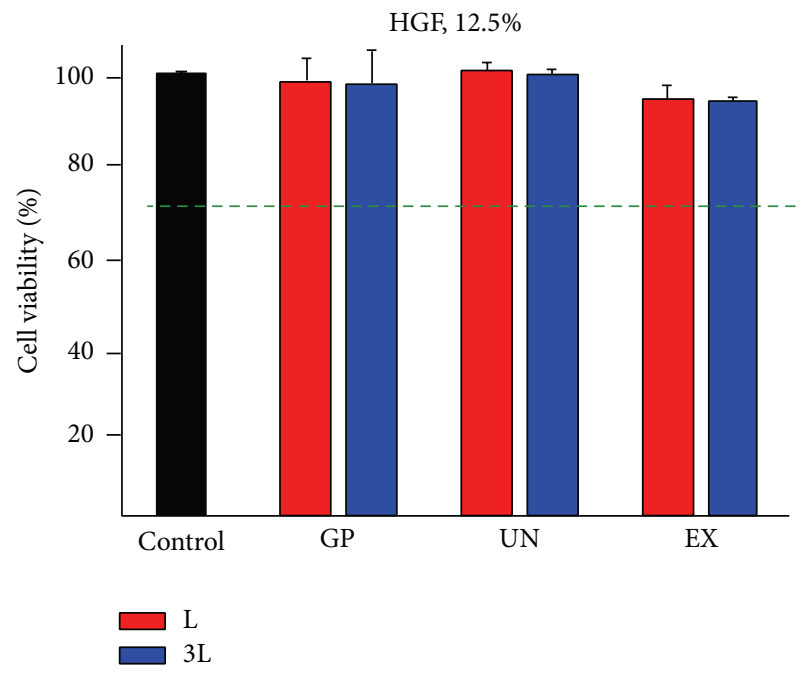

(c)

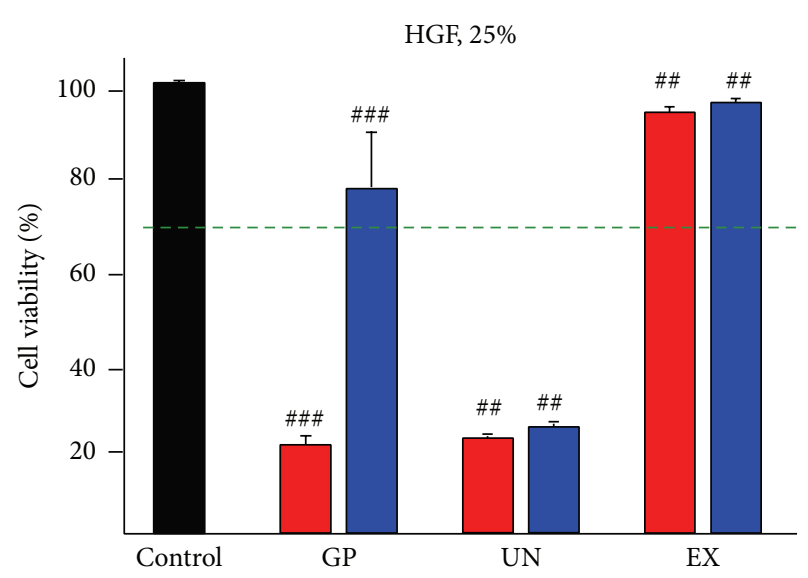

(b)

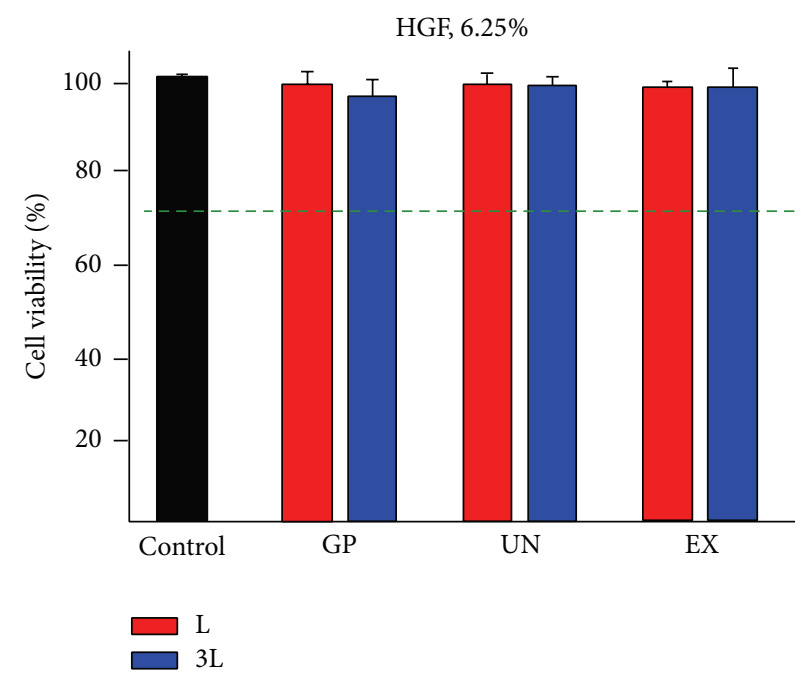

(d)

FIGURE 2: Human gingival fibroblast (HGF) viability following exposure to the extracts from the "3L" and " $\mathrm{L}$ " latex rubber bands of GP, UN, and EX. The following dilutions were used: (a) $50 \%$; (b) 25\%; (c) 12.5\%; (d) $6.25 \%$. The difference in the cell viability was statically determined under a few experimental conditions $\left(n=6,{ }^{\# \#} P<0.01,{ }^{\# \#} P<0.001\right.$; between the "3L" and "L" extracts of each evaluated product). 3L: stretched to three times its length, L: original length. Representative results are shown after the experiments were performed in triplicate.

TABLE 3: The concentration of the elements in the samples (\%).

\begin{tabular}{lcccc}
\hline Sample & Carbon & Hydrogen & Nitrogen & Sulfur \\
\hline GP-L & $10.81 \pm 0.27$ & $2.31 \pm 0.04$ & $1.60 \pm 0.14$ & $0.39 \pm 0.04$ \\
GP-3L & $10.49 \pm 1.18$ & $2.23 \pm 0.19$ & $1.54 \pm 0.04$ & $0.38 \pm 0.05$ \\
UN-L & $9.78 \pm 1.19$ & $2.16 \pm 0.29$ & $1.19 \pm 0.19$ & $0.15 \pm 0.08$ \\
UN-3L & $9.76 \pm 0.35$ & $2.06 \pm 0.09$ & $1.15 \pm 0.06$ & $0.12 \pm 0.02$ \\
EX-L & $10.11 \pm 0.55$ & $2.47 \pm 0.32$ & $1.20 \pm 0.13$ & $0.49 \pm 0.11$ \\
EX-3L & $10.09 \pm 1.20$ & $2.16 \pm 0.26$ & $1.21 \pm 0.16$ & $0.24 \pm 0.13$ \\
Control & $9.78 \pm 0.67$ & $2.21 \pm 0.16$ & $1.21 \pm 0.17$ & $0.98 \pm 0.19$ \\
\hline
\end{tabular}

Concentration of the "L" and " $3 \mathrm{~L}$ " samples from the GP, UN, and EX extracts.

a cell lysis zone extending from the specimen up to $1 \mathrm{~cm}$. According to ISO 10993-12, natural rubber latex was used for the positive control [16]. Therefore, the "3L" and "L" groups had moderate cytotoxicity due to their strong cytotoxicity. The E group had a reduced cell lysis zone compared with the
GP and UN groups, which showed similar results from the cytotoxicity test to those of the L929 cells and HGFs.

To explain the difference in the viability between the "L" and "3L" groups, the following assumption was made. Cytotoxic particles may rapidly detach from the latex rubber 


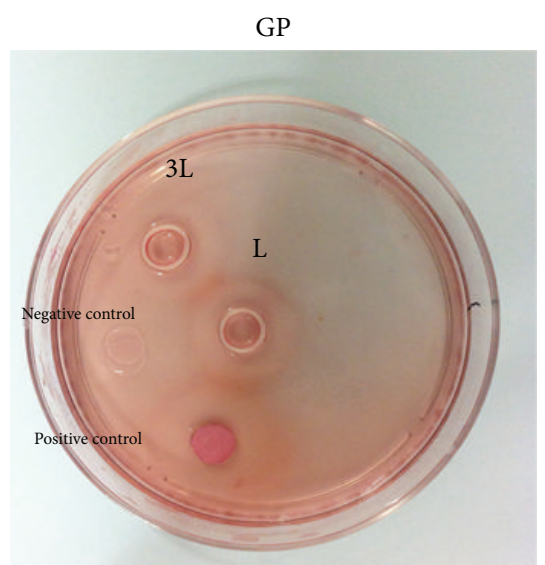

(a)

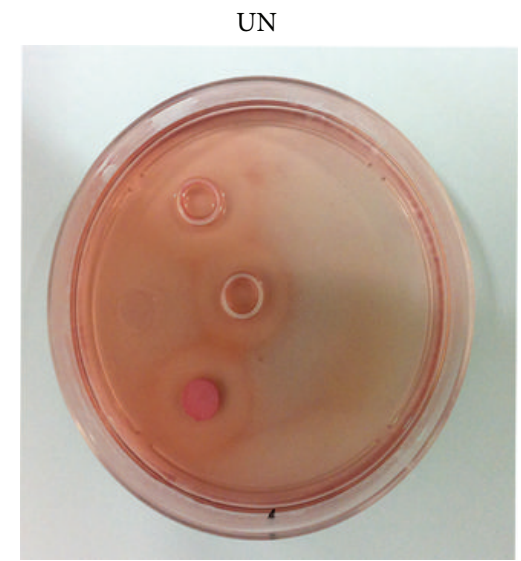

(b)

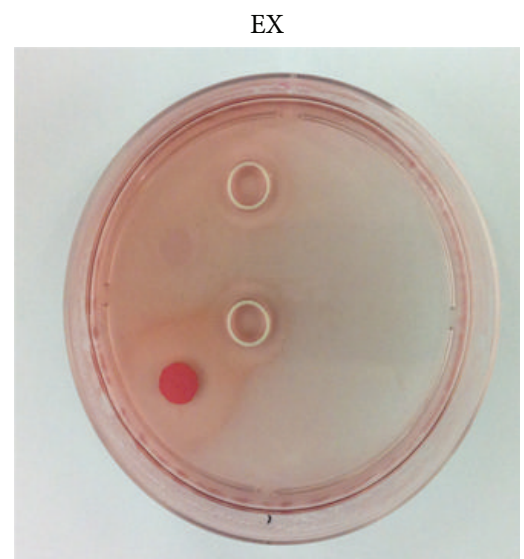

(c)

FIgURE 3: Agar diffusion test. The latex sheet from the latex glove (positive control), the high density polyethylene sheet (negative control), and "L" and " $3 \mathrm{~L}$ " of (a) GP, (b) UN, and (c) EX were located in predetermined positions. A zone extending up to $1.0 \mathrm{~cm}$ around the specimen boundary was detected in all rubber band groups. Representative images are shown after the experiments were performed in triplicate.

TABLE 4: The concentration of the elements in the latex rubber bands (ppm).

\begin{tabular}{lccccc}
\hline Sample & Fluoride & Chloride & Nitrate & Phosphate & Sulfate \\
\hline GP-L & $17.64 \pm 0.07$ & $3992.08 \pm 3.24$ & $41.42 \pm 0.16$ & $444.57 \pm 3.12$ & $30.35 \pm 0.27$ \\
GP-3L & $17.83 \pm 0.17$ & $3986.18 \pm 1.50$ & $42.15 \pm 0.13^{*}$ & $450.52 \pm 2.39$ & $30.89 \pm 0.19$ \\
UN-L & $18.16 \pm 0.07^{*}$ & $4003.26 \pm 7.87$ & $42.87 \pm 0.60$ & $459.9 \pm 7.69$ & $31.57 \pm 0.48^{*}$ \\
UN-3L & $17.81 \pm 0.05$ & $3990.32 \pm 7.05$ & $41.45 \pm 0.84$ & $441.48 \pm 8.24$ & $30.34 \pm 0.57$ \\
EX-L & $17.29 \pm 0.11$ & $3984.58 \pm 21.52$ & $40.13 \pm 0.99$ & $429.54 \pm 4.96$ & $29.32 \pm 0.71$ \\
EX-3L & $17.35 \pm 0.21$ & $3990.82 \pm 5.66$ & $40.33 \pm 0.81$ & $432.47 \pm 7.59$ & $29.49 \pm 0.60$ \\
Control & $17.75 \pm 0.34$ & $3973.25 \pm 15.07$ & $41.73 \pm 0.90$ & $455.83 \pm 9.61$ & $30.36 \pm 0.64$ \\
\hline
\end{tabular}

Concentration of the "L" and " $3 \mathrm{~L}$ " samples from the GP, UN, and EX extracts.

${ }^{*} P<0.05$ compared with the control and $3 \mathrm{~L}$ sample in each product.

band surface to the air when the bands are stretched up to three times because the length increased outside of the extracting media, whereas the attached cytotoxic particles of the "L" group, which may have an increased amount of attached harmful materials, may be released more from the latex rubber bands when incubated as "L" without stretching.

To reveal the potentially harmful components and show the cytotoxicity difference between the " $\mathrm{L}$ " and " $3 \mathrm{~L}$ " extracts, FT-IR measurements were initially used to characterize the extracts. Previous studies have suggested that the cytotoxicity of elastic bands may be due to preservatives, such as zinc oxide and sulfur (S), and due to the presence of an activator, including nickel compounds and hydroquinone, which are known cytotoxic substances [29]. In this study, the FTIR transmittance measurements with the ATR device were performed to detect the presence of harmful components from the extracts of the " $3 \mathrm{~L}$ " and "L" groups. The FT-IR results indicated that opaque minerals (native metal, nickel, zinc, or a metallic oxide mineral) might be present (see Figure 4, 1020, 2850, and $2917 \mathrm{~cm}^{-1}$ ) [30] without any significant difference between the "L" and " $3 \mathrm{~L}$ " groups, which was considered due to the limitation of the reflectance mode of FT-IR when ATR and the media extract were used for the evaluation [31].
To determine the presence of a cytotoxic inducer, the inner and outer surfaces of the latex rubber bands were investigated using SEM images with EDS elemental analysis of the "L" and "3L" groups. On the outer surface, $\mathrm{Zn}$ was detected as the major cytotoxic inducer in the latex rubber bands in all the products (data not shown). The $\mathrm{Zn}$ ion could be extracted from $\mathrm{ZnO}$, a preservative used in latex rubber bands, and has been considered as a key factor in the induction of cytotoxicity $[9,11]$. In accordance with previous studies, the results of the ICP-MS showed that the $\mathrm{Zn}$ ion increased in all the extract groups compared with the control culture media, which indicated that the latex bands stored under wet conditions may elute the cytotoxic cation, as found in other studies that evaluated in vitro cytotoxicity [32]. The concentration of the $\mathrm{Zn}$ ion ( $9 \mathrm{ppm})$ from the extracts was higher than the initiating cytotoxicity level $(5 \mathrm{ppm})$, indicating that the $\mathrm{Zn}$ ion was one of the key inducers of cytotoxicity [33]. However, other possible inducers were not excluded due to the severe cytotoxicity of the latex rubber bands. The $\mathrm{Zn}$ ion was detected at a lower concentration in the " $3 \mathrm{~L}$ " extracts than in the "L" extracts of the $\mathrm{P}$ and $\mathrm{E}$ groups, which suggested a detachment of the other deposited components. Therefore, other components from the latex rubber bands could be considered as cytotoxic inducers. 


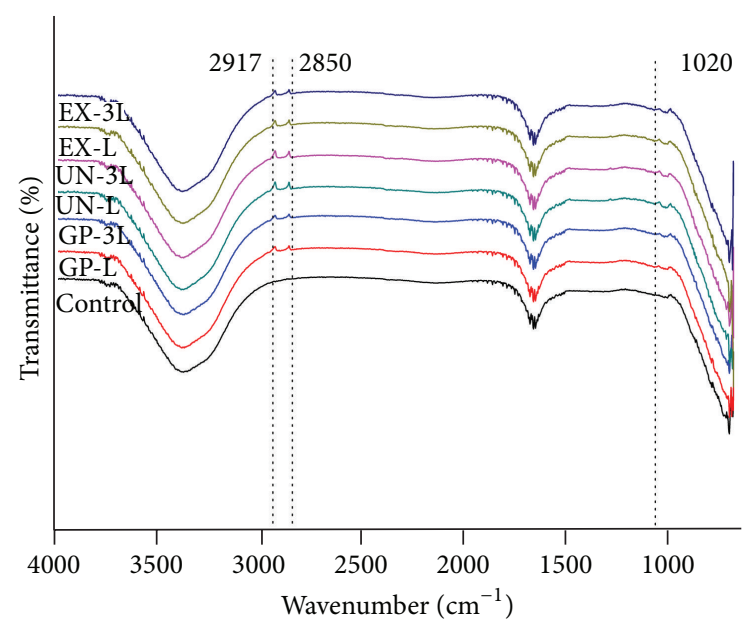

FIGURE 4: FT-IR spectra of the latex rubber band extracts. The DMEM (control) and the extracts of the P, U, and E groups for the "L" and stretched " $3 \mathrm{~L}$ " samples. The results of the FT-IR indicated an increased IR-transmittance at 1020 (C-O stretch), 2850, and $2917 \mathrm{~cm}^{-1}$ (C-H stretch) compared with the DMEM culture media (control group), which indicated the presence of opaque minerals in the extracts, such as native metal, zinc, or a metallic oxide mineral. Representative images were shown after the experiments were performed in triplicate.

On the inner surface, many detachable particles were observed, and the "L" group had more attached particles than the "3L" group for all the products (Figures 5 and 6). The clearing of Ni nanoparticles was only detected in the "3L" group but not in the "L" group for all the products according to the EDS results, whereas sulfur (S), one of the possible cytotoxic ingredients, was detected at the same level (Figure 6). Ion chromatography and elemental analysis were used to additionally detect any differences in the sulfate and sulfide contents among the "L" group, "3L" group, and culture media because researchers have mentioned that sulfur-related materials may be potentially cytotoxic $[6,29]$. Unfortunately, no difference was detected (Tables 3 and 4). The clearing of the Ni single crystal nanoparticles from the inner surface of the latex rubber bands was supported by the EDS mapping results in Figure 7 and the TEM images with the SEAM pattern in Figure 8. However, the $\mathrm{Ni}$ ion was not detected by ICP-MS in the control and experimental groups due to its low ionization in extracting media. The cytotoxicity of the Ni nanoparticles was more severe than that of the $\mathrm{Ni}$ oxide nanoparticles, which induced cytotoxicity from 400 ppm [34] compared with the low concentration ( 2 ppm) of the Ni nanoparticles $[35,36]$. There has been concern that the unique characteristics of the nanomaterials themselves induce undesirable effects despite the absence of heavy metals in the nanomaterials. However, the concentration above the cytotoxicity-inducing level was determined to be more than a few hundred ppm, which is significantly higher than the concentration of the extract from the latex rubber bands [37]. Thus, the "3L" extracts would show a lower cytotoxicity due to the clearing of the highly toxic Ni nanoparticles along with the decrease in the extracted toxic $\mathrm{Zn}$ ions [38].
Ni nanoparticles are included in the vulcanizing processing of latex rubber bands. Uncured natural latex rubber deforms easily under warm conditions and is brittle when cold, which makes it a poor material when a high level of elasticity is required. Vulcanization of the latex rubber, the chemical process to convert the natural rubber into a more durable material via the addition of sulfur, results in crosslinking via the disulfide bonds among the natural rubbers, which prevents the long polymer chains in the rubber from moving independently and consequently increases the elasticity [39]. During vulcanization, activators are essential ingredients, which reduce the curing time by increasing the rate of vulcanization. The common activators used are nickel compounds, zinc oxide, hydroquinone, phenol, alphanaphthylamine, and P-phenylenediamine, which have been considered to be cytotoxic inducers [40]. In addition to the cytotoxicity of the activator, these compounds have been widely used in other latex materials $[41,42]$. Therefore, the deposited Ni nanoparticles are an inevitable phenomenon in the process of vulcanization of latex rubber.

Latex rubber bands are widely used in clinical applications, although they showed cytotoxicity in in vitro tests. The in vitro cytotoxicity may be attenuated when the latex rubber bands are used in vivo due to the dynamic saliva circulation compared with the static test conditions of the in vitro tests. Furthermore, according to the results of this study, the stretching motion of the latex rubber bands outside or within the mouth aids in the rapid detachment of the cytotoxic materials, decreasing the release of the cytotoxic element to the oral mucosa. The results of the cytotoxicity test depend on the cell type used in the experiment [43]. Although human oral epithelial cells, components of the outer layer in the oral mucosa, might have been a better choice to mimic the harmful effect on the oral mucosa, the cytotoxicity results of the mouse L929 cells and HGFs, inner components of the oral mucosa, provided insight into the cytotoxic effect of the latex rubber bands due to their vulnerability to the cytotoxic inducer [44].

Stretching of the latex rubber bands caused a decrease in the cytotoxicity, which appears to be more relevant to the clinical outcome in which it is relatively a less harmful event to the patients due to the detachment of $\mathrm{ZnO}$ and the Ni nanoparticles when using the latex rubber bands in an orthodontic treatment. Previously, the cytotoxic effect of the deposited particles covering latex elastics has been questioned [18]. According to this study, the $\mathrm{ZnO}$ and $\mathrm{Ni}$ nanoparticles covering the latex rubber bands could be cytotoxic inducers in the latex rubber bands. The presented results supported the assumption that cytotoxic Ni nanoparticles on the latex rubber bands may rapidly detach from the inner surface to the air when the bands are stretched up to three times their length, contributing to the safe usage of the latex rubber bands in orthodontic treatments, regardless of the in vitro cytotoxicity.

\section{Conclusions}

The "3L" group showed a different cytotoxicity in the L929 and HGF cells compared with the "L" group due to the 

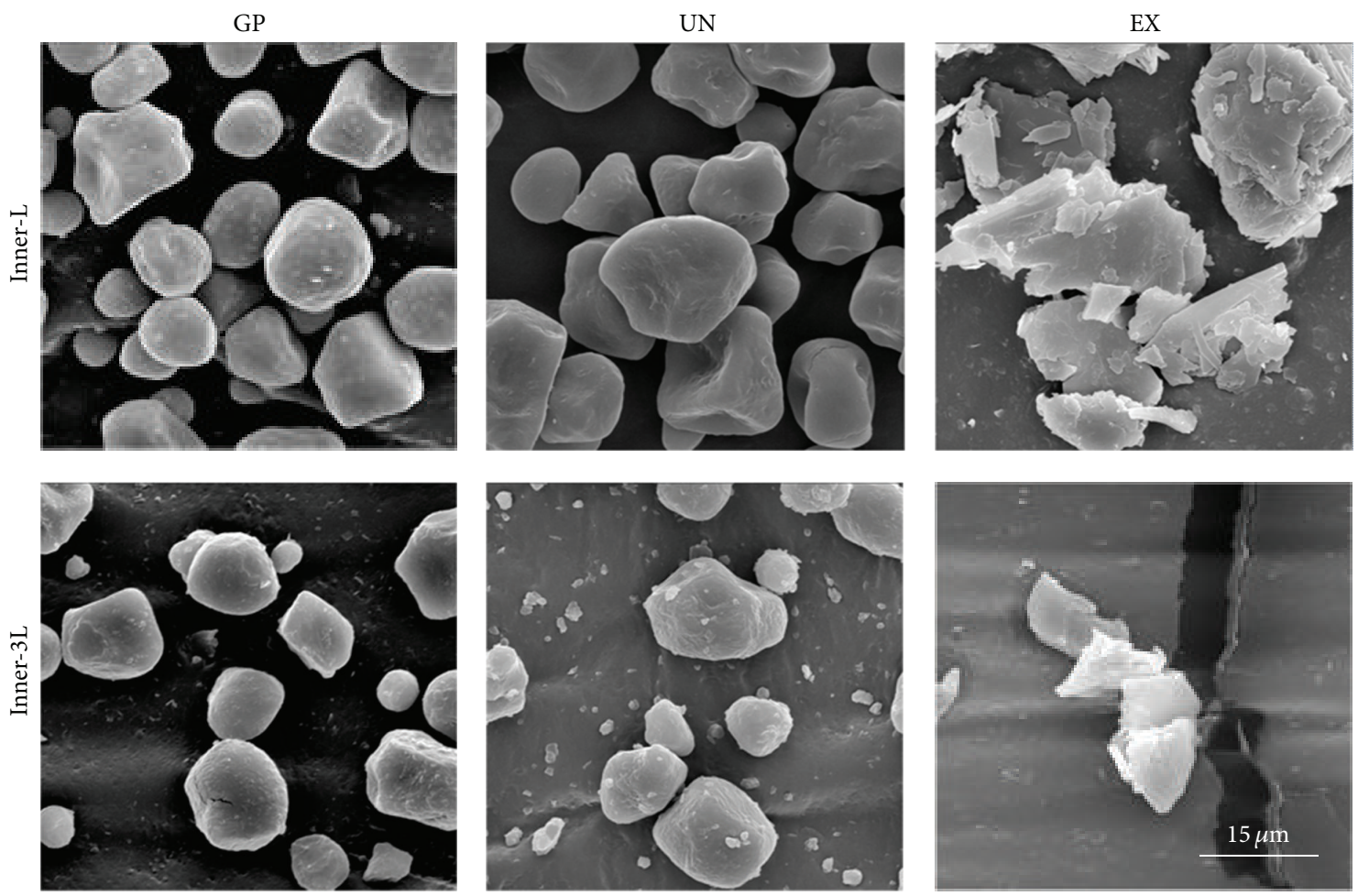

(a)
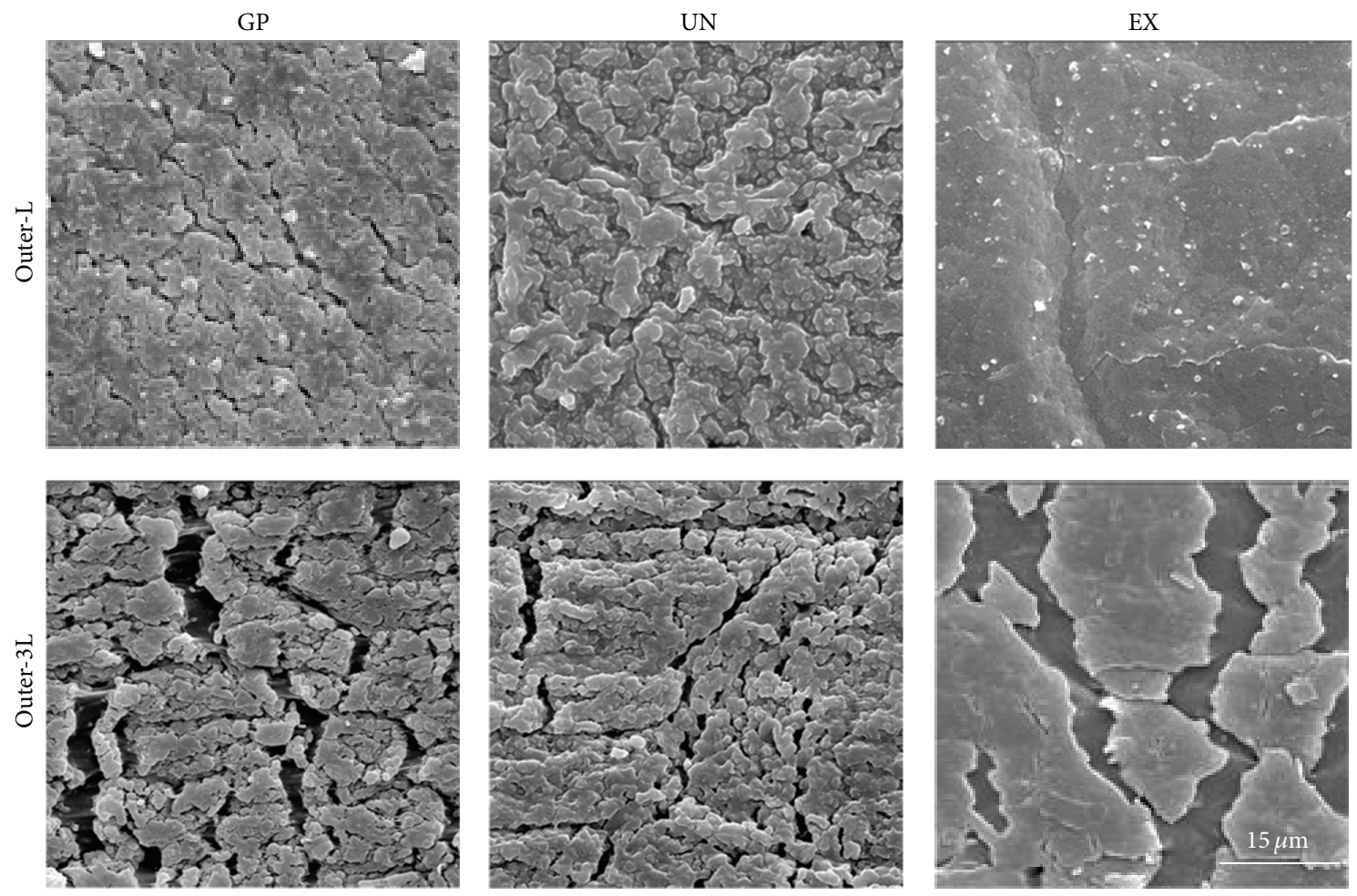

(b)

FIGURE 5: SEM images of the "L" and " $3 \mathrm{~L}$ " rubber band inner and outer surfaces. The deposited particles of the "L" group were reduced in the "3L" group on the inner surface. However, on the outer surface, there were few attached particles in both the "L" and " $3 \mathrm{~L}$ " groups compared with the inner surface, and a crevice was detected in the "3L" group. Representative images were shown after the experiments were performed in triplicate. 

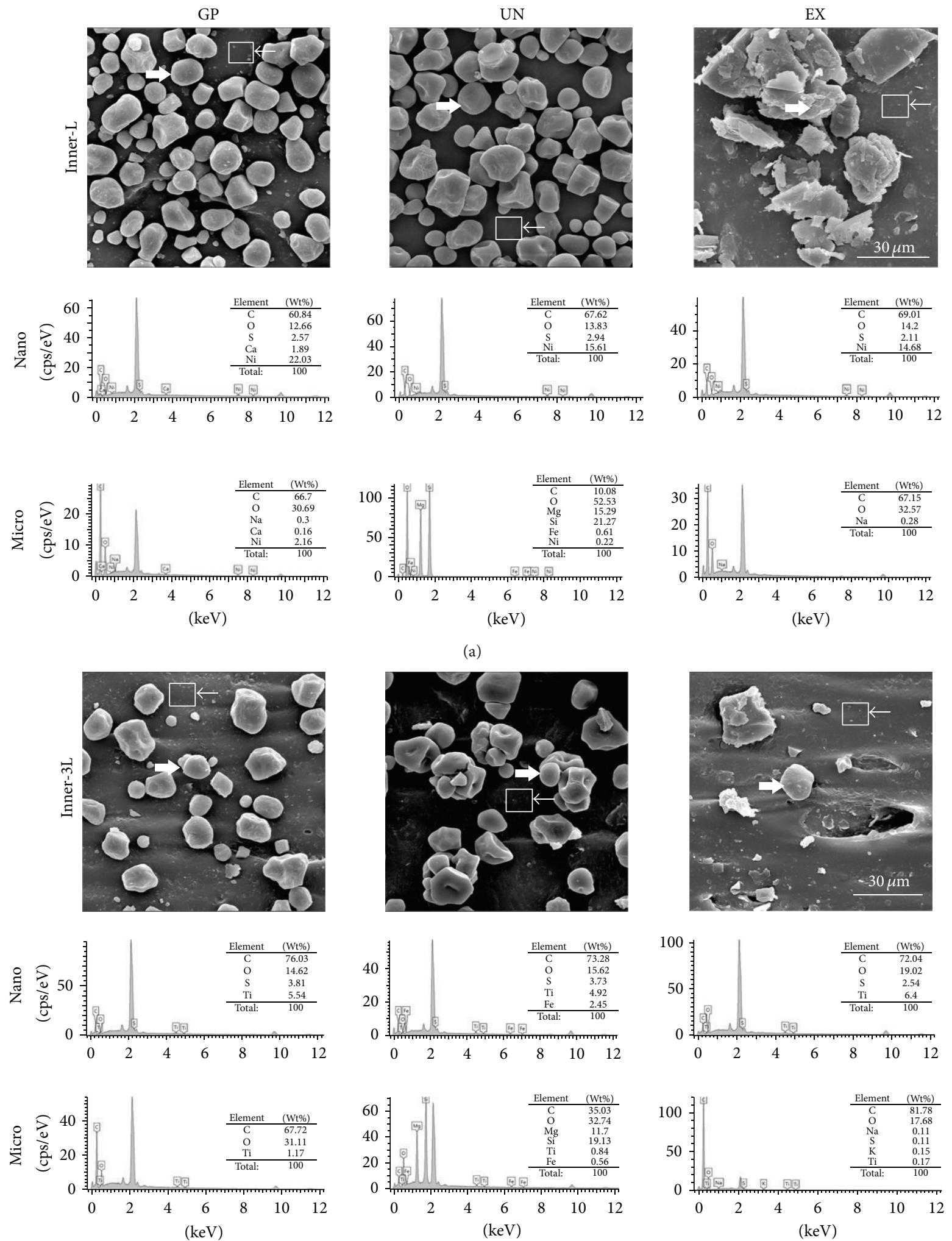

(b)

FIGURE 6: SEM images and elemental analysis using EDS from the (a) "L" and (b) "3L" rubber band inner surface. The left headed white arrow and the right headed arrow with a rectangle indicate the microsized particles and the nanosized particles, respectively. A decrease in the microsized particles $(\sim 30 \mu \mathrm{m})$ and a disappearance of the nickel nanosize particles $(\sim 50 \mathrm{~nm})$ were observed in the Inner-3L. Representative images were shown after the experiments were performed in triplicate. 
GP

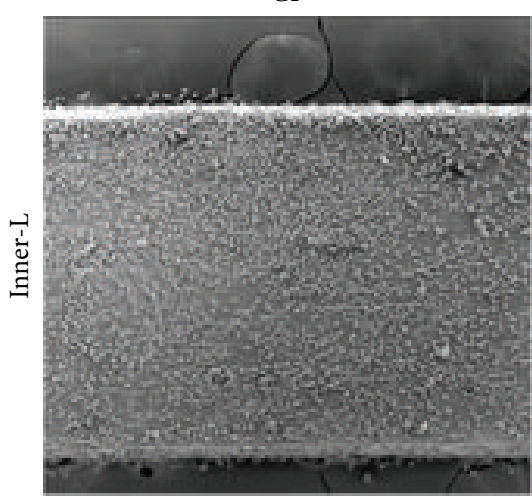

(a)

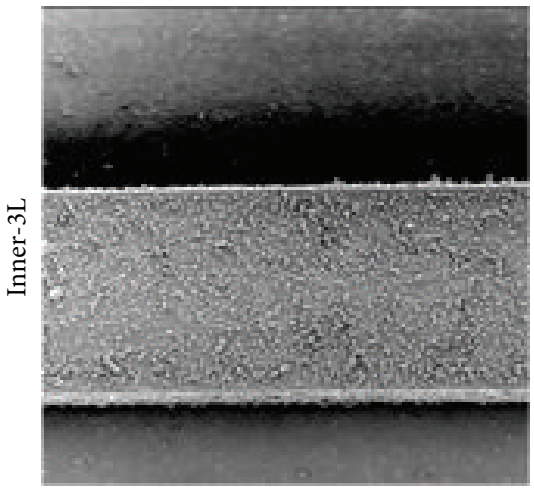

(d)

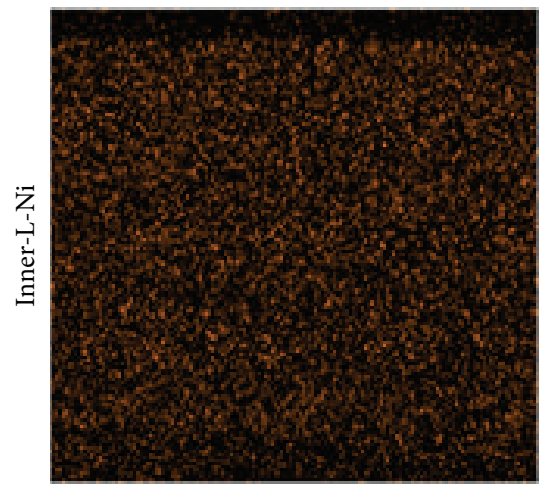

(g)
UN

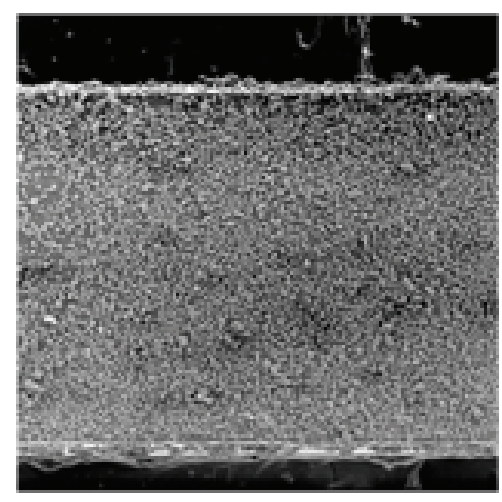

(b)

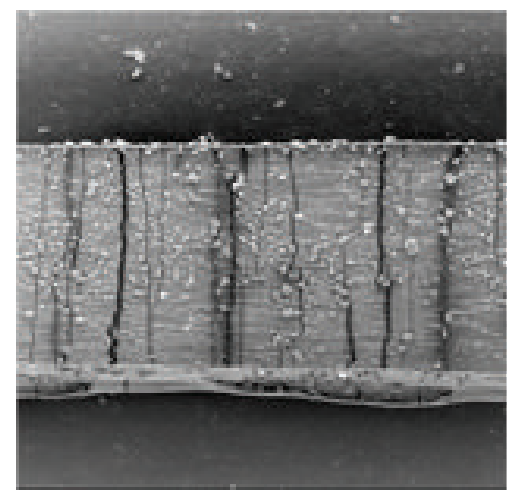

(e)

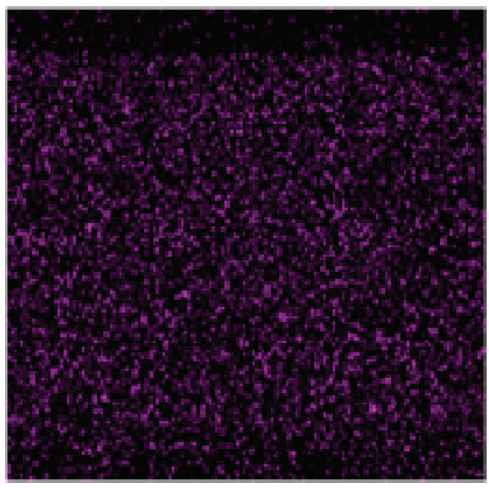

(h)

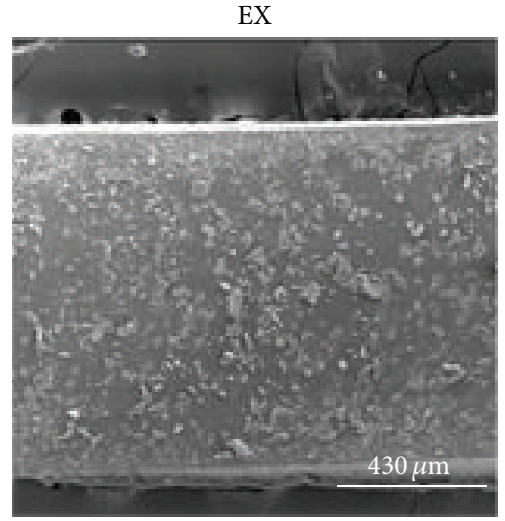

(c)

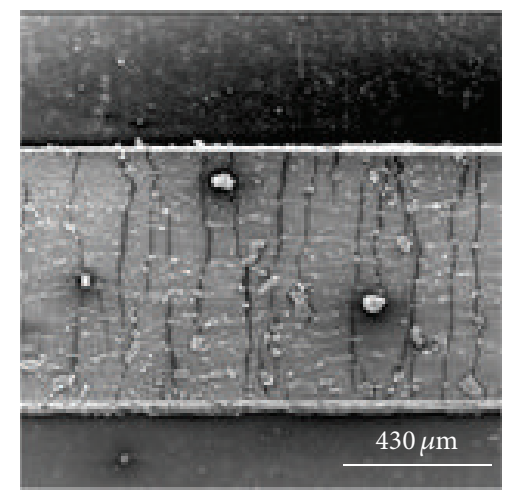

(f)

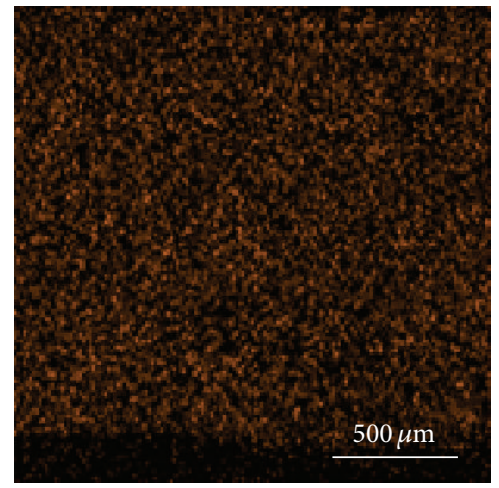

(i)

FIGURE 7: SEM images of the "L" ((a), (b), and (c)) and "3L" ((d), (e), and (f)) rubber band inner surfaces. A decrease in the attached particles was observed in the "3L" group. The colored dots on the "Inner-L-Ni" ((g), (h), and (i)) show the presence of nickel nanoparticles on the "L". However, nickel nanoparticles on the "3L" were not detected (images cannot be obtained). Representative images were shown after the experiments were performed in triplicate.

detachment of the $\mathrm{ZnO}$ preservative and the Ni nanoparticles, which are inevitably used in the vulcanization process of the latex rubber. These results appear to be relevant to the safe usage of the latex rubber bands in orthodontic treatments due to the detachment of the harmful particles. The stretching procedure prior to the use of latex rubber bands in orthodontic treatments could be the process during which the potential nanoparticles detach from the surface.

\section{Abbreviations}

L: $\quad$ Original length

3L: $\quad$ Stretched to three times

HGF: Human gingival fibroblast

FT-IR: Fourier transform infrared spectrometry

ATR: Attenuated total reflectance

ICP-MS: Inductively coupled plasma mass spectrometry 

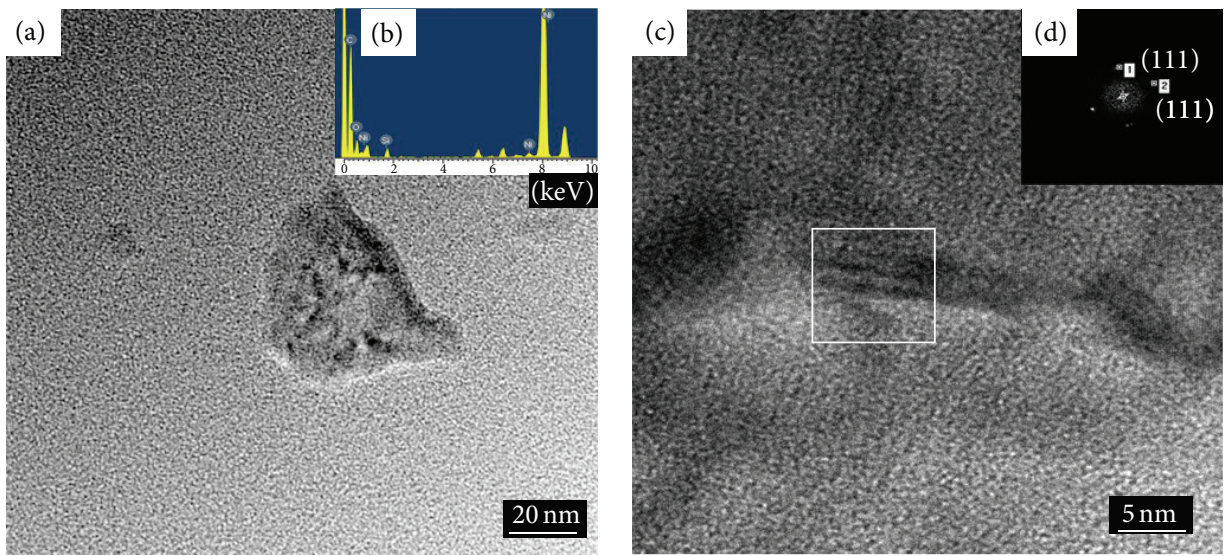

FIgURE 8: TEM images of the detached nanoparticles from the GP product with the SEAD pattern images. The TEM image of the Ni nanoparticles was observed at a size of $50 \mathrm{~nm}$ in (a). The insert (b) shows the EDS results, which confirmed the presence of nickel in the nanoparticle. (c) HR-TEM image of the nanoparticle. The insert (d) shows the SAED pattern along the (111) zone axis, which confirmed the single crystal structure of nickel. Representative images were shown after the experiments were performed in triplicate.

SEM: Scanning electron microscope

EDS: Energy dispersive spectroscopy

TEM: Transmission electron microscopy

SAED: Selected area electron diffraction.

\section{Conflict of Interests}

The authors declare that there is no conflict of interests regarding the publication of this paper.

\section{Authors' Contribution}

Chung-Ju Hwang and Kyoung-Nam Kim equally organized this study as corresponding authors.

\section{Acknowledgment}

This research was supported by a Grant (12172KFDA501) from the Korea Food and Drug Administration in 2012.

\section{References}

[1] A. Kunzmann, B. Andersson, T. Thurnherr, H. Krug, A. Scheynius, and B. Fadeel, "Toxicology of engineered nanomaterials: focus on biocompatibility, biodistribution and biodegradation," Biochimica et Biophysica Acta, vol. 1810, no. 3, pp. 361-373, 2011.

[2] L. Zhang, Y. Hong, T. Zhang, and C. Li, "A novel approach to prepare $\mathrm{PBT}$ nanocomposites with elastomer-modified $\mathrm{SiO}_{2}$ Particles," Polymer Composites, vol. 30, no. 5, pp. 673-679, 2009.

[3] M. Hannig and C. Hannig, "Nanomaterials in preventive dentistry," Nature Nanotechnology, vol. 5, no. 8, pp. 565-569, 2010.

[4] W. Song, J. Wang, and M. Liu, "Titanium dioxide nanoparticles induced proinflammation of primary cultured cardiac myocytes of rat," Journal of Nanomaterials, vol. 2013, Article ID 349140, 9 pages, 2013.

[5] X. Li, S. C. Lee, S. Zhang, and T. Akasaka, "Biocompatibility and toxicity of nanobiomaterials 2013," Journal of Nanomaterials, vol. 2014, Article ID 821293, 2 pages, 2014.
[6] W. Boonchai, P. Iamtharachai, P. Kasemsarn, and W. Sirikudta, "Latex glove allergy among health care workers: verification by testing for both type I and type IV hypersensitivity reactions," Journal of the American Academy of Dermatology, vol. 66, no. 4, Supplment 1, p. AB73, 2012.

[7] S. Graumüller, F. Schwarz, B. Kramp, D. Mallon, and H. W. Paul, "Prevalence of IgE-mediated natural rubber latex allergies in health care workers (HCW) in Rostock, Germany, and Fremantle Hospital, Perth, Australia," Allergologie, vol. 27, no. 3, pp. 87-94, 2004.

[8] N. López, A. Vicente, L. A. Bravo, J. L. Calvo, and M. Canteras, "In vitro study of force decay of latex and non-latex orthodontic elastics," The European Journal of Orthodontics, vol. 4, no. 2, pp. 202-207, 2011.

[9] C. Hwang and J. Cha, "Mechanical and biological comparison of latex and silicone rubber bands," American Journal of Orthodontics and Dentofacial Orthopedics, vol. 124, no. 4, pp. 379-386, 2003.

[10] R. L. dos Santos, M. M. Pithon, F. O. Martins, M. T. V. Romanos, and A. C. de Oliveira Ruellas, "Evaluation of the cytotoxicity of latex and non-latex orthodontic separating elastics," Orthodontics and Craniofacial Research, vol. 13, no. 1, pp. 28-33, 2010.

[11] M. M. Pithon, R. L. dos Santos, F. O. Martins, M. T. V. Romanos, and M. T. D. Araújo, "Cytotoxicity of orthodontic separating elastics," Australian Orthodontic Journal, vol. 26, no. 1, pp. 1620, 2010.

[12] J. Holmes, M. K. Barker, E. K. Walley, and O. C. Tuncay, "Cytotoxicity of orthodontic elastics," American Journal of Orthodontics and Dentofacial Orthopedics, vol. 104, no. 2, pp. 188-191, 1993.

[13] R. L. dos Santos, M. M. Pithon, P. P. de Melo Freire, and M. T. V. Romanos, "In vitro study of cytotoxicity of orthodontic elastomeric ligatures," Materials Research, vol. 15, no. 4, pp. 657661, 2012.

[14] V. G. Kokich, "In-vitro vs in-vivo materials research," The American Journal of Orthodontics and Dentofacial Orthopedics, vol. 143, no. 4, p. S11, 2013.

[15] International Standard Organization (ISO), ISO 10993-5 Biological Evaluation of Medical Devices-Part 5: Tests for In Vitro Cytotoxicity, ISO, Geneva, Switzerland, 2009. 
[16] International Standard Organization (ISO), ISO 10993-12 Biological Evaluation of Medical Devices-Part 12: Sample Preparation and Reference Materials, ISO, Geneva, Switzerland, 3rd edition, 2007.

[17] International Standard Organization (ISO), ISO 21606 Dentistry-Elastomeric Auxiliaries for Use in Orthodontics, ISO, Geneva, Switzerland, 2007.

[18] R. L. dos Santos, M. M. Pithon, and M. T. V. Romanosc, "The influence of $\mathrm{pH}$ levels on mechanical and biological properties of nonlatex and latex elastics," The Angle Orthodontist, vol. 82, no. 4, pp. 709-714, 2012.

[19] J. S. Kwon, S. B. Lee, C. K. Kim, and K. N. Kim, "Modified cytotoxicity evaluation of elastomeric impression materials while polymerizing with reduced exposure time," Acta Odontologica Scandinavica, vol. 70, no. 6, pp. 597-602, 2012.

[20] F. Öztürk, S. Malkoc, M. Ersöz, S. S. Hakki, and B. S. Bozkurt, "Real-time cell analysis of the cytotoxicity of the components of orthodontic acrylic materials on gingival fibroblasts," The American Journal of Orthodontics and Dentofacial Orthopedics, vol. 140, no. 5, pp. E243-E249, 2011.

[21] G. R. Casaccia, J. C. Gomes, D. S. Alviano, A. C. de Oliveira Ruellas, and E. F. Sant'Anna, "Microbiological evaluation of elastomeric chains," The Angle Orthodontist, vol. 77, no. 5, pp. 890893, 2007.

[22] A. David and D. Lobner, "In vitro cytotoxicity of orthodontic archwires in cortical cell cultures," The European Journal of Orthodontics, vol. 26, no. 4, pp. 421-426, 2004.

[23] B. S. McAllister, F. Leeb-Lundberg, and M. S. Olson, "Bradykinin inhibition of EGF- and PDGF-induced DNA synthesis in human fibroblasts," The American Journal of Physiology-Cell Physiology, vol. 265, no. 2, part 1, pp. C477-C484, 1993.

[24] R. L. Gerlach and T. N. Rhodin, "Structure analysis of alkali metal adsorption on single crystal nickel surfaces," Surface Science, vol. 17, no. 1, pp. 32-68, 1969.

[25] W. Ahmed, R. P. B. Laarman, C. Hellenthal, E. S. Kooij, A. van Silfhout, and B. Poelsema, "Dipole directed ring assembly of Nicoated Au-nanorods," Chemical Communications, vol. 46, no. 36, pp. 6711-6713, 2010.

[26] S. B. Mitra, "Nanoparticles for dental materials: synthesis, analysis, and applications," in Emerging Nanotechnologies in Dentistry, K. Subramani and W. Ahmed, Eds., chapter 2, pp. 15-33, William Andrew, Boston, Mass, USA, 2012.

[27] K. L. van Landuyt, K. Yoshihara, B. Geebelen et al., "Should we be concerned about composite (nano-)dust?" Dental Materials, vol. 28, no. 11, pp. 1162-1170, 2012.

[28] K. L. van Landuyt, B. Hellack, B. van Meerbeek et al., "Nanoparticle release from dental composites," Acta Biomaterialia, vol. 10, no. 1, pp. 365-374, 2014.

[29] W. Fiddler, J. Pensabene, J. Sphon, and D. Andrzejewski, "Nitrosamines in rubber bands used for orthodontic purposes," Food and Chemical Toxicology, vol. 30, no. 4, pp. 325-326, 1992.

[30] M. A. Ziemann and V. Lüders, "Characterization of IR-transmittance of sulfides and other opaque minerals by FTIR-spectroscopy," in Proceedings of the 6th Biennial Pan-American Conference on Research on Fluid Inclusions, P. E. Brown and S. G. Hagemann, Eds., pp. 149-151, Madison, Wis, USA, 1996.

[31] PerkinElmer Life and Analytical Sciences, "FT-IR Spectroscopy-Attenuated Total Reflectance (ATR)," Perkin Elmer Life and Analytical Sciences, Shelton, 2005.

[32] L. J. Zhang, X. Q. Mu, J. L. Fu, and Z. C. Zhou, "In vitro cytotoxicity assay with selected chemicals using human cells to predict target-organ toxicity of liver and kidney," Toxicology in Vitro, vol. 21, no. 4, pp. 734-740, 2007.

[33] W. Song, J. Zhang, J. Guo et al., "Role of the dissolved zinc ion and reactive oxygen species in cytotoxicity of $\mathrm{ZnO}$ nanoparticles," Toxicology Letters, vol. 199, no. 3, pp. 389-397, 2010.

[34] K. Ada, M. Turk, S. Oguztuzun et al., "Cytotoxicity and apoptotic effects of nickel oxide nanoparticles in cultured HeLa cells," Folia Histochemica et Cytobiologica, vol. 48, no. 4, pp. 524-529, 2010.

[35] M. Ahamed, "Toxic response of nickel nanoparticles in human lung epithelial A549 cells," Toxicology in Vitro, vol. 25, no. 4, pp. 930-936, 2011.

[36] C. Ispas, D. Andreescu, A. Patel, D. V. Goia, S. Andreescu, and K. N. Wallace, "Toxicity and developmental defects of different sizes and shape nickel nanoparticles in zebrafish," Environmental Science \& Technology, vol. 43, no. 16, pp. 6349-6356, 2009.

[37] T. Yoshida, Y. Yoshioka, K. Matsuyama et al., "Surface modification of amorphous nanosilica particles suppresses nanosilicainduced cytotoxicity, ROS generation, and DNA damage in various mammalian cells," Biochemical and Biophysical Research Communications, vol. 427, no. 4, pp. 748-752, 2012.

[38] S. Lanone, F. Rogerieux, J. Geys et al., "Comparative toxicity of 24 manufactured nanoparticles in human alveolar epithelial and macrophage cell lines," Particle and Fibre Toxicology, vol. 6, no. 1, article 14, 2009.

[39] H.-W. Engels, H.-J. Weidenhaupt, M. Pieroth et al., "Rubber, 4. Chemicals and additives," in Ullmann's Encyclopedia of Industrial Chemistry, Wiley-VCH, 2000.

[40] B. Sengupta, Development of Standards for Ubber Products Manufacturing Industry, Central Pollution Control Board Ministry of Environment \& Forests, Delhi, India, 2007.

[41] L. G. Wideman, "Process for hydrogenation of carbon-carbon double bonds in an unsaturated polymer in latex form," Google Patents, 1984.

[42] D. K. Parker and D. M. Ruthenburg, "Process for the preparation of hydrogenated rubber," Google Patents, 1995.

[43] H. Lang and T. Mertens, "The use of cultures of human osteoblastlike cells as an in vitro test system for dental materials," Journal of Oral and Maxillofacial Surgery, vol. 48, no. 6, pp. 606611, 1990.

[44] K. Moharamzadeh, R. van Noort, I. M. Brook, and A. M. Scutt, "Cytotoxicity of resin monomers on human gingival fibroblasts and HaCaT keratinocytes," Dental Materials, vol. 23, no. 1, pp. 40-44, 2007. 

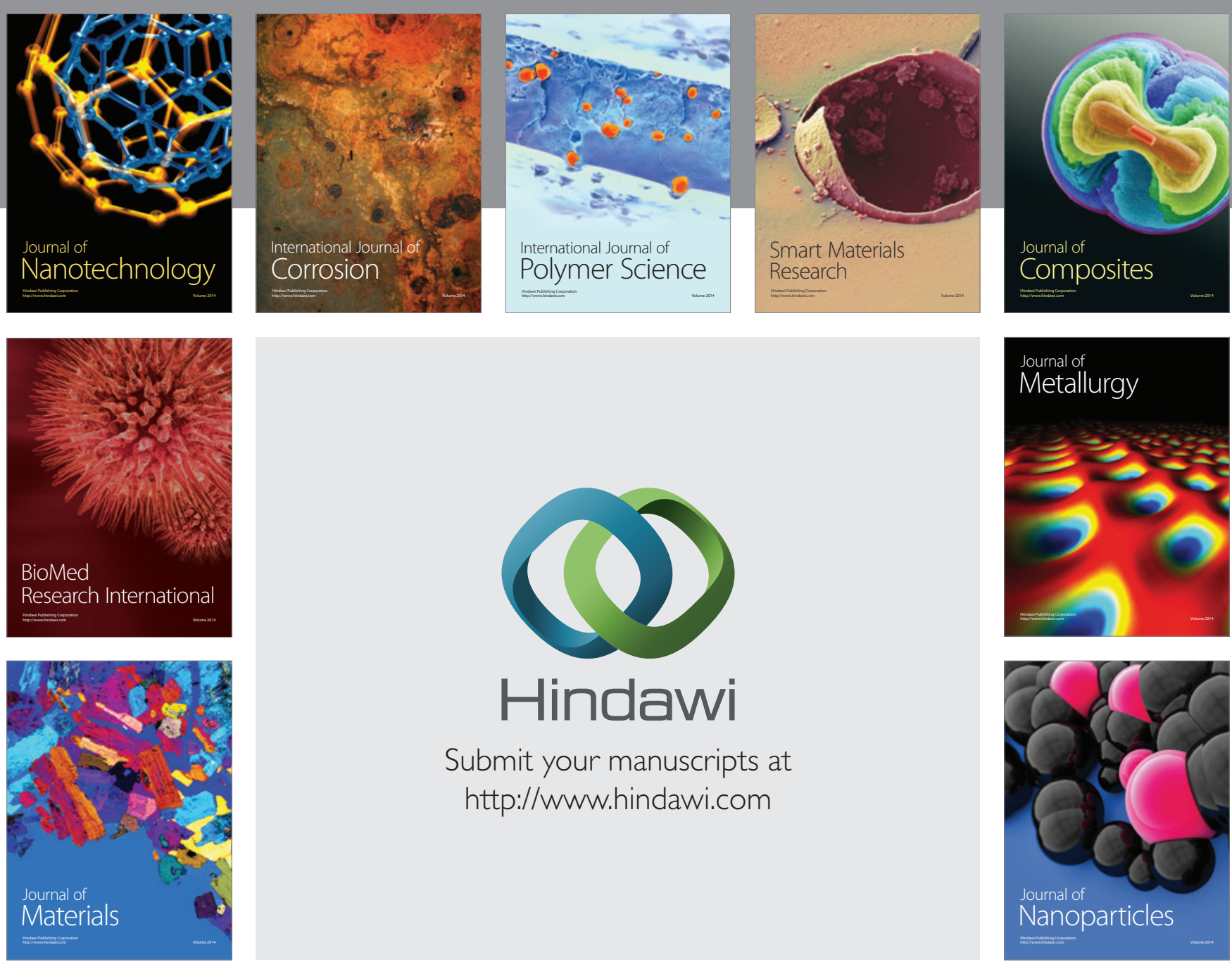

Submit your manuscripts at http://www.hindawi.com
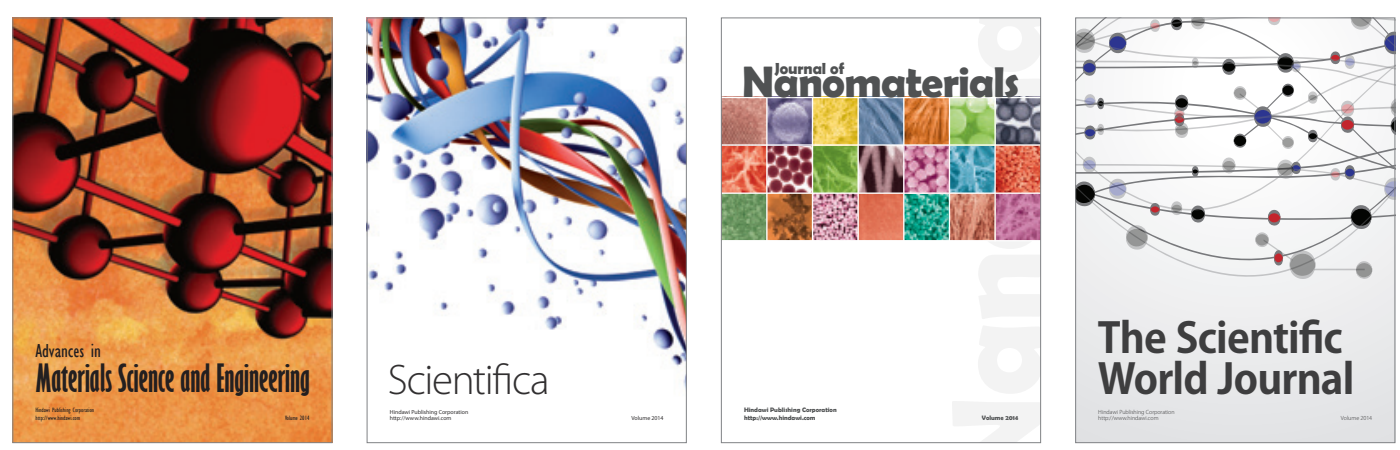

\section{The Scientific World Journal}
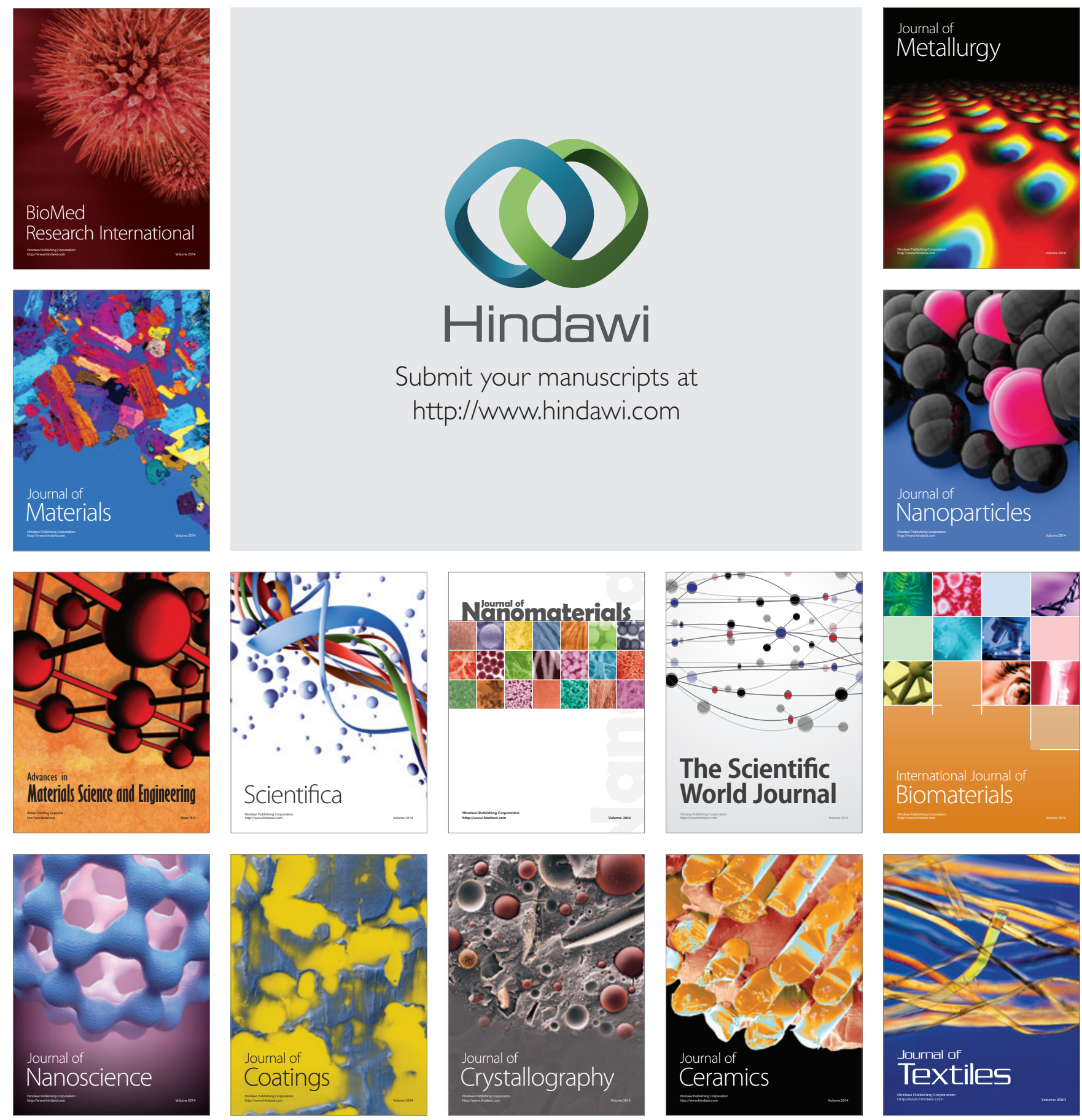